Reprod. Nutr. Dévelop., 1982, 22 (6), 959-971.

\title{
Mélatonine dans l'organe pinéal, la rétine et le plasma. Etude immunologique chez le Pigeon
}

\author{
P. VOISIN, M. GEFFARD $\left({ }^{*}\right)$, M. DELAAGE $\left({ }^{*}\right)$, J.-P. COLLIN
}

Laboratoire de Zoologie et Biologie cellulaire

(Equipe de Neurobiologie et Neuroendocrinologie cellulaires), et L.A. CNRS $n^{\circ} 290$,

40, avenue du Recteur-Pineau, 86022 Poitiers Cedex, France.

${ }^{*}$ ) Institut de Biochimie cellulaire et Neurochimie,

1, rue Camille-Saint-Saëns, 33000 Bordeaux, France.

Summary. An immunological study of melatonin in the pineal organ, retina and plasma of the pigeon.

Circadian variations in melatonin content have been studied in the pineal organ, retina and plasma of adult pigeons using radioimmunoassay. A clear-cut peak of melatonin was observed in the pineal organ at middark; this middark peak was of larger amplitude in July than in November. A nocturnal rise in melatonin concentration was also observed in the retina and plasma.

We also used an immunocytochemical technique revealing the positive reactions of a melatonin-like compound in the photoreceptor cell layer (inner segments) of the pigeon's retina. Up to now, no melatonin storage has been visualized in the pineal organ of the pigeon.

\section{Introduction.}

Des expériences de physiologie du comportement réalisées chez le Moineau (Gaston et Menaker, 1968 ; Zimmerman et Menaker, 1979) ont conduit leurs auteurs à conclure que l'organe pinéal joue un rôle primordial dans l'organisation de l'activité locomotrice de l'Oiseau selon un rythme circadien, propriété qui a pu être rattachée au métabolisme indolique, après la découverte de l'effet synchronisant de l'injection de mélatonine chez des animaux pinéalectomisés arythmiques (Gwinner et Benzinger, 1978). Par contre, le rôle éventuel de la mélatonine dans le contrôle de la fonction de reproduction, de même que ses sites de synthèse et de stockage dans l'épiphyse et autres organes et tissus sont encore insuffisamment connus chez l'Oiseau (revue de Ralph, 1981). En vue d'établir, chez le Pigeon, certaines des propriétés de ce méthoxyindole dans la régulation neuroen-

Correspondance: Dr. Pierre Voisin, Laboratoire de Zoologie et Biologie Cellulaire, 40, avenue du Recteur-Pineau, 86022 Poitiers Cedex, France. 
docrinienne, nous comparons, en été et en automne, ses variations nycthémérales. Chez cette espèce, seul le rythme circadien de la sérotonine (5-HT), précurseur de la mélatonine et celui de l'acide-5-hydroxyindole acétique (5-HIAA) ont été étudiés antérieurement dans les conditions de laboratoire (Quay, 1966).

Grâce aux progrès réalisés dans la production d'anticorps dirigés contre des molécules de faible poids moléculaire et l'introduction de dosages radioimmunologiques de la mélatonine (réf. in Rollag, 1981 ; Geffard et al., 1982), il est maintenant possible de doser cette molécule sur une seule épiphyse, voire même sur des fractions de l'organe. Compte tenu des analogies qui existent entre les cellules de la lignée réceptrice de l'épiphyse (Collin et Oksche, 1981) et les cônes et bâtonnets de la rétine, nous avons effectué une étude parallèle du cycle circadien de la mélatonine dans ces deux structures. Les variations nycthémérales du taux plasmatique de la mélatonine ont également été envisagées. La forte affinité de l'anticorps et sa haute spécificité pour la mélatonine (Geffard et al., 1982) nous ont incité à l'employer en vue de l'identification - par immunohistochimie - des cellules impliquées dans le stockage de cet indole.

\section{Matériel et méthodes.}

\section{1. - Animaux.}

Des Pigeons domestiques (Columba livia, Gmelin) des deux sexes (de 12 à 18 mois ; 400-500 g) sont utilisés pour le dosage radioimmunologique de la mélatonine. Les conditions d'élevage ont été précisées antérieurement (Voisin, 1982 a, b). Les Oiseaux sont ici conditionnés selon le cycle d'éclairement naturel (Poitiers, France). Une première série expérimentale est réalisée au mois de juillet. La photopériode $(16 \mathrm{~L} / 8 \mathrm{D})$ est de $16 \mathrm{~h}$ de lumière $(=\mathrm{L}$ : à partir de $6 \mathrm{~h}$ du matin) et $8 \mathrm{~h}$ d'obscurité $(=\mathrm{D})$. Une deuxième série expérimentale est utilisée en novembre (régime photopériodique : 10L/14D ; phase claire à partir de $8 \mathrm{~h}$ du matin).

Des Pigeons sont également sacrifiés au cours de la séquence sombre, en vue de l'identification des sites de stockage de la mélatonine par immunohistochimie.

\section{2. - Techniques.}

a) Traitement pharmacologique. - La parachlorophenylalanine (p-CPA : AB-Biotec, Göteborg, Sweden) inhibiteur de la tryptophane hydroxylase (Koe and Weissman, 1966) est utilisée pour inhiber la synthèse de la 5-HT et par conséquent celle de la mélatonine. Les animaux reçoivent, par voie intrapéritonéale, trois injections de p-CPA à la dose de $300 \mathrm{mg} / \mathrm{kg}$ (48 h, $24 \mathrm{~h}$, et $12 \mathrm{~h}$ avant le sacrifice).

b) Anticorps utilisé et principe du RIA. - L'anticorps dirigé contre la mélatonine, utilisé ici, a été obtenu par Geffard et al. (1982). Cet anticorps est produit chez le Lapin après injection d'un immunogène réalisé par le branchement d'un 
haptène (la N-succinyl-5-méthoxytryptamine) sur la sérum albumine humaine. La spécificité antigénique est portée par l'haptène dont ta structure est très voisine de celle de la mélatonine.

L'anticorps obtenu reconnaît très spécifiquement la mélatonine ; le taux de croisement le plus élevé (1 p. 100) concerne la 5-méthoxytryptophol. La forte affinité de l'anticorps pour la mélatonine ( $\left.\mathrm{Kd}=2 \cdot 10^{-9} \mathrm{M}\right)$ permet de doser cette molécule avec une bonne précision dans une gamme de concentrations allant de $10^{-10} \mathrm{M}$ à $10^{-8} \mathrm{M}$.

Le traceur radioactif est obtenu par la fixation de la N-succinyl-5méthoxytryptamine sur le dipeptide glycyl-tyrosine, suivie d'une iodation du radical hydroxyphényl de la tyrosine par l'iode 125.

Le dosage radioimmunologique est effectué par dialyse à l'équilibre (Geffard et al., 1982).

\section{c) Dosage de la mélatonine.}

- Sacrifice. Dissection. - Les animaux sont sacrifiés très rapidement (décapitation) par groupe de trois, toutes les $2 \mathrm{~h}$ pendant $24 \mathrm{~h}$.

- Expérience / (prélèvements réalisés au mois de juillet). - Les épiphyses sont homogénéisées au sonificateur dans $600 \mu \mathrm{l}$ de tampon phosphate $0,1 \mathrm{M}$, $\mathrm{pH}=7,2$ contenant 0,1 p. 100 de gélatine. Après centrifugation pendant $3 \mathrm{~min}$ à $10000 \mathrm{~g}$, le surnageant est séparé et conservé à $-30^{\circ} \mathrm{C}$ jusqu'au moment des analyses. Des aliquots de $200 \mu \mathrm{l}$ sont prélevés auxquels on ajoute $400 \mu \mathrm{l}$ de dioxane. Après agitation au vortex et centrifugation on obtient une sédimentation des protéines. Le surnageant contient la mélatonine (le rendement de l'extraction est constant et proche de $100 \mathrm{p}$. 100). Le surnageant est congelé pendant une nuit à $-80^{\circ} \mathrm{C}$ puis lyophilisé pendant $24 \mathrm{~h}$. Le lyophilisat est repris dans $200 \mu \mathrm{l}$ d'eau bidistillée. Cette solution sera utilisée pour les RIA.

Au moment de la décapitation, 2 à $5 \mathrm{ml}$ de sang sont prélevés dans un tube hépariné. Après centrifugation à $2000 \mathrm{~g}$ pendant $10 \mathrm{~min}$, le surnageant est prélevé et congelé à $-30^{\circ} \mathrm{C}$.

A des aliquots de $400 \mu \mathrm{l}$ de plasma on ajoute $800 \mu \mathrm{l}$ de dioxane. Après centrifugation, $10000 \mathrm{~g}$ pendant $15 \mathrm{~min}$, le surnageant est prélevé, congelé et lyophilisé. Le lyophilisat est repris dans $400 \mu$ d'eau distillée.

- Expérience // (prélèvements réalisés au mois de novembre). - Aussitôt prélevées, les épiphyses et les rétines sont congelées dans l'azote liquide, puis stockées à $-30^{\circ} \mathrm{C}$.

L'extraction de la mélatonine est réalisée en homogénéisant les échantillons dans $500 \mu \mathrm{l}$ d'un mélange dioxane/eau distillée : 2/1. Après centrifugation, $10000 \mathrm{~g}$ pendant $15 \mathrm{~min}$, le surnageant est séparé du culot protéique et congelé à :- $80^{\circ} \mathrm{C}$ pendant une nuit avant d'être lyophilisé pendant $24 \mathrm{~h}$. Le lyophilisat est repris dans $500 \mu \mathrm{l}$ d'eau bidistillée. Le radio-immuno-essai sera appliqué sur cette solution. Les culots protéiques des rétines sont conservés pour le dosage des protéines selon la technique de Lowry et al. (1951). Le rendement d'extraction de la mélatonine est équivalent selon les deux méthodes. 
- Radio-immuno-essai par dialyse à l'équilibre. - Des aliquots de $200 \mu \mathrm{lde}$ la solution aqueuse de mélatonine reçoivent un volume égal d'une solution de dérivé iodé. Chaque chambre à dialyse reçoit dans un compartiment $150 \mu$ de l'anticorps à la dilution $1 / 200000^{e}$, dans l'autre $150 \mu$ l du mélange traceur iodé et solution à quantifier.

La dialyse se déroule à $4^{\circ} \mathrm{C}$ pendant $22 \mathrm{~h}$ sous agitation continue. Passé ce délai, l'équilibre est atteint ; le contenu de chaque compartiment est prélevé et sa radioactivité est mesurée dans un compteur $\gamma$ (Intertechnique).

d) Immunohistochimie. - Les animaux sont sacrifiés au milieu de la phase obscure, entre décembre et février. Les épiphyses et les rétines sont congelées à - $30{ }^{\circ} \mathrm{C}$ ou fixées au glutaraldéhyde à 2 p. 100 dans du tampon phosphate $0,1 \mathrm{M}, \mathrm{pH}=7,2$, isotonique (PBS) et incluses dans le glycol-méthacrylate (GMA), selon la méthode de Leduc et Bernhard (1967).

- Préparation des coupes de tissus congelés. - Les tissus congelés sont enrobés dans du cryoform (Damon/IEC Division) et sectionnés au microtome cryostat (Harris, WRC).

Les coupes de $10 \mu \mathrm{m}$ d'épaisseur déposées sur lame porte-objet sont fixées par l'éthanol $100^{\circ}$ (à $-25^{\circ} \mathrm{C}$, pendant 2 s) ou par le glutaraldéhyde (1 p. 100 dans le PBS) à $4{ }^{\circ} \mathrm{C}$, pendant $1 \mathrm{~min}$. Les lames sont ensuite rincées dans le PBS pendant $15 \mathrm{~min}$.

- Préparation des coupes de tissus inclus dans le GMA. - Des coupes de $1 \mu \mathrm{m}$ d'épaisseur sont obtenues sur l'ultramicrotome Reichert OMU 2 et déposées sur lames porte-objet.

\section{- Traitement immunologique.}

- Tre étape : Le sérum natif contenant l'anticorps anti-mélatonine est dilué au $1 / 10^{\mathrm{e}}, 1 / 25^{\mathrm{e}}, 1 / 50^{\mathrm{e}}$ et $1 / 100^{\mathrm{e}}$, dans le tampon PBS. Ces solutions sont déposées sur les coupes. Les préparations témoins sont mises en contact avec un sérum de Lapin anti-immunoglobulines de Cobaye (SLAC) (Institut Pasteur Production) dilué au $1 / 10^{\mathrm{e}}$ et $1 / 100^{\mathrm{e}}$. L'incubation est effectuée en chambre humide et à l'obscurité pendant $1 \mathrm{~h}$, à $37^{\circ} \mathrm{C}$ ou pendant $24 \mathrm{~h}$ à la température du laboratoire. Passé ce délai, les préparations subissent deux rinçages de 10 min dans le tampon PBS.

- $2^{e}$ étape : Les coupes sont alors mises en présence d'un sérum de Mouton, anti-immunoglobuline de Lapin, marqué par l'isothiocyanate de fluorescéine (Institut Pasteur Production). Cet anticorps est dilué au $1 / 10^{\mathrm{e}}$ dans du tampon PBS contenant 0,01 p. 100 de Bleu Evans. L'incubation se déroule dans les mêmes conditions que précédemment pendant $1 \mathrm{~h}$ à $37^{\circ} \mathrm{C}$. Les coupes sont rincées dans le PBS, montées dans de la glycérine tamponnée et observées au microscope Leitz "Orthoplan », équipé d'une lampe à vapeur de mercure (Osram HBO 200 W) pour excitation dans I'ultraviolet. 


\section{Résultats.}

1. - Variations quantitatives circadiennes et saisonnières de la mélatonine.

a) Dans l'épiphyse. - La mesure des variations circadiennes du taux de mélatonine dans l'épiphyse permet de mettre en évidence une augmentation de la concentration de cet indole en phase obscure (fig. 1 et 2).

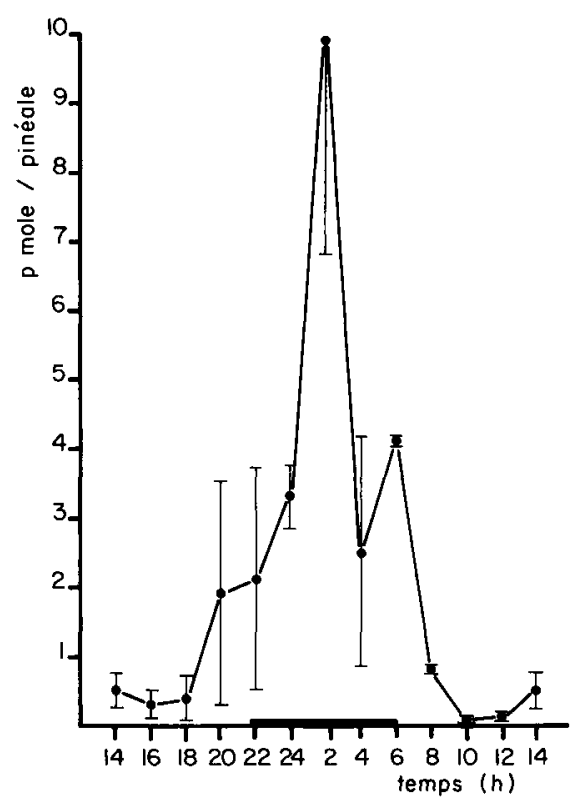

FIG. 1.

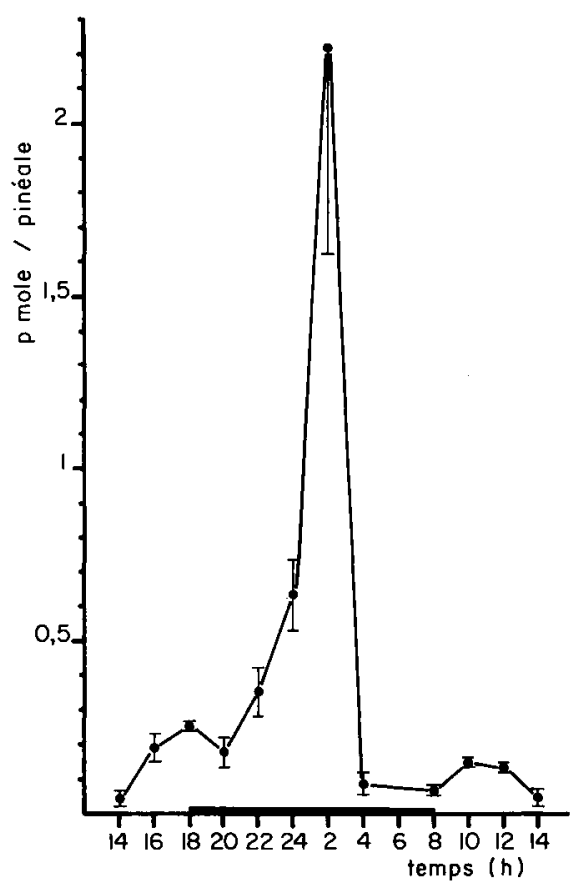

FIG. 2 .

FIG. 1. - Variations nycthémérales de la mélatonine dans l'épiphyse du Pigeon. Dosages effectués au mois de juillet. L/D : 16/8 - Début de la phase claire : environ 6 h du matin. Les concentrations sont exprimées en pmole/pinéale (moyenne \pm écart-type, $n=3$ ).

FIG. 2. - Variations nycthémérales de la mélatonine dans l'épiphyse du Pigeon. Dosages effectués au mois de novembre. L/D : 10/14 - Début de la phase claire : environ $8 \mathrm{~h}$ du matin. Les concentrations sont exprimées en pmole/pinéale (moyenne \pm écart-type, $n=3$ ).

Quelle que soit l'expérience considérée (| ou II : voir Matériel et méthodes) le pic est situé à $02: 00$ et est significatif par rapport aux concentrations mesurées à $24: 00$ et à $04: 00$ (expérience $1: p<0,02$; expérience $\mathrm{II}: \mathrm{p}<0,01$; test $t$ de Student). Les variations saisonnières se traduisent au niveau de l'amplitude du pic par une valeur nettement plus élevée en juillet qu'en novembre. Le rapport entre la concentration au pic et la concentration moyenne en période diurne, est deux fois plus élevé en juillet qu'en novembre. 
b) Dans la rétine. - Des variations individuelles importantes dans certains groupes de mesures ne permettent pas de décrire précisément le cycle nycthéméral de la mélatonine rétinienne. Cependant, l'analyse des données par l'épreuve de la médiane généralisée permet de conclure que la concentration en mélatonine est plus élevée au cours de la phase sombre que pendant la phase claire (tabl. 1).

\section{TABLEAU 1}

Fluctuations circadiennes de la mélatonine en novembre (Analyse des données par l'épreuve de la médiane généralisée)

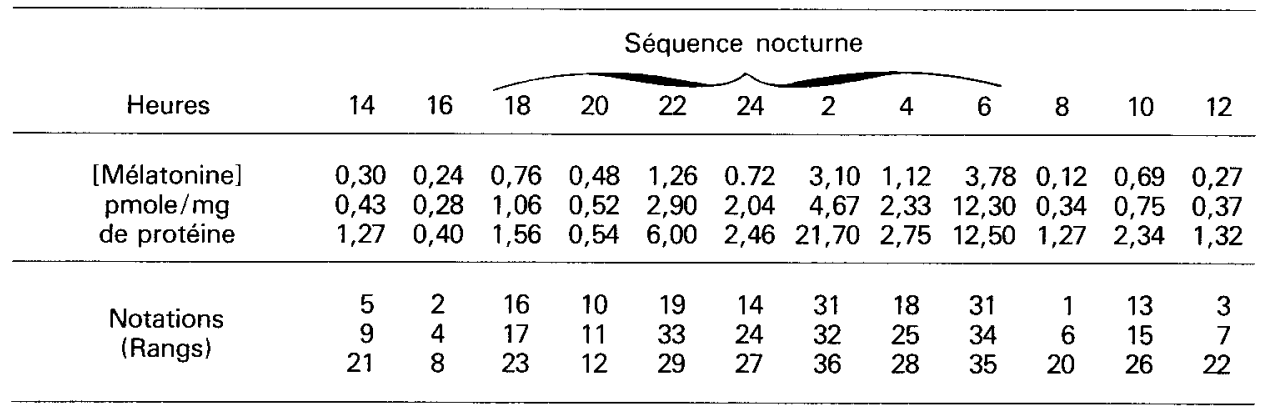

La médiane se situe entre 18 et 19

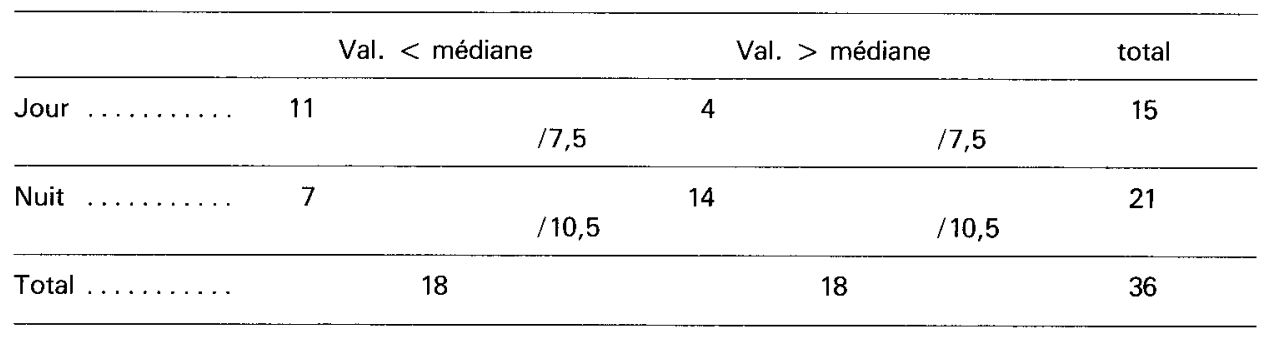

$$
\chi^{2}=5,58 \text { (significatif à } p<0,05 \text { ) }
$$

c) Dans le plasma. - Une augmentation importante de la concentration plasmatique de la mélatonine est observée au cours de la séquence sombre de la photopériode (fig. 3). La moyenne des valeurs enregistrées à $24: 00$ et à $04: 00$ est 10 fois supérieure à celle des valeurs mesurées entre $08: 00$ et $20: 00$.

\section{2. - Sites de stockage de la mélatonine.}

a) Dans l'épiphyse. - Malgré de nombreuses tentatives, il n'a pas été possible jusqu'ici de visualiser les sites de stockage de la mélatonine dans l'épiphyse du Pigeon. Une fluorescence aspécifique intense affecte l'épithélium épiphysaire et les méninges après traitement des préparations par l'anticorps anti-mélatonine 
ou le sérum témoin. L'incubation préalable des préparations dans une solution de gélatine à 0,75 p. 100 permet de diminuer la fluorescence aspécifique, mais aucune réaction pouvant traduire la présence de mélatonine n'est alors observable dans les tissus.

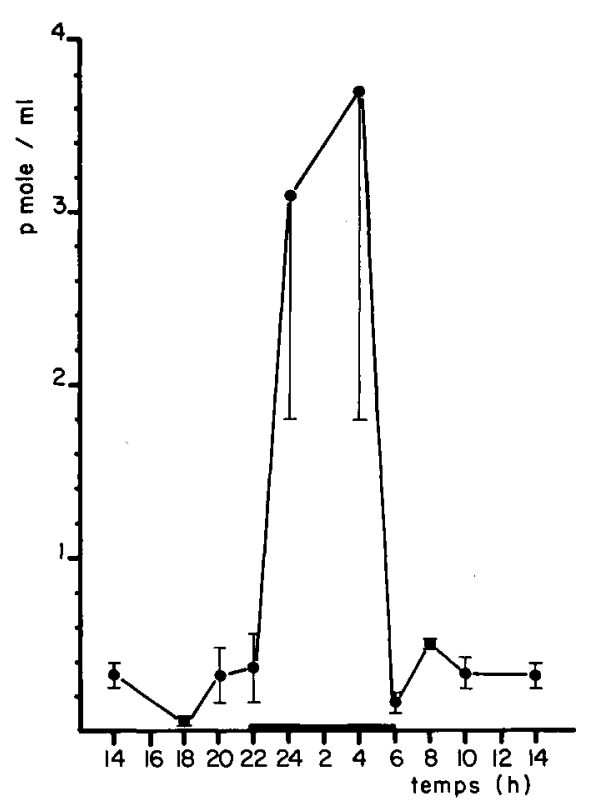

FIG. 3. - Variations nycthémérales de la concentration plasmatique de la mélatonine chez le Pigeon. Dosages effectués au mois de juillet. Les concentrations sont exprimées en pmole $/ \mathrm{ml}$ (moyenne \pm écart-type, $\mathrm{n}=3$ ).

b) Dans la rétine. - Les expériences d'immunofluorescence réalisées chez le Pigeon sur des rétines sectionnées au cryostat, donnent des résultats constants. Les produits de la réaction s'observent régulièrement au niveau de la couche nucléaire externe (fig. 4), lorsque le premier anticorps est utilisé à de faibles dilutions $\left(1 / 10^{\mathrm{e}}\right)$; ils diminuent parallèlement à la concentration de l'anticorps jusqu'à disparaître (dilution $1 / 50^{\mathrm{e}}$ ). Les réactions observées semblent donc traduire la fixation du premier anticorps et non une réaction aspécifique de l'anticorps marqué (utilisé à concentration constante). Aucune fluorescence n'est observable sur les préparations témoins où un SLAC remplace l'anticorps dirigé contre la mélatonine (fig. 5).

Lorsque le métabolisme indolique est interrompu à l'étape de l'hydroxylation du tryptophane par le traitement des animaux par la p-CPA, on observe une diminution sensible de la fluorescence au niveau de la couche nucléaire externe (fig. 6).

Sur les rétines fixées au glutaraldéhyde et incluses dans le GMA, une fluorescence très intense apparaît dans une bande contiguë à la couche nucléaire 

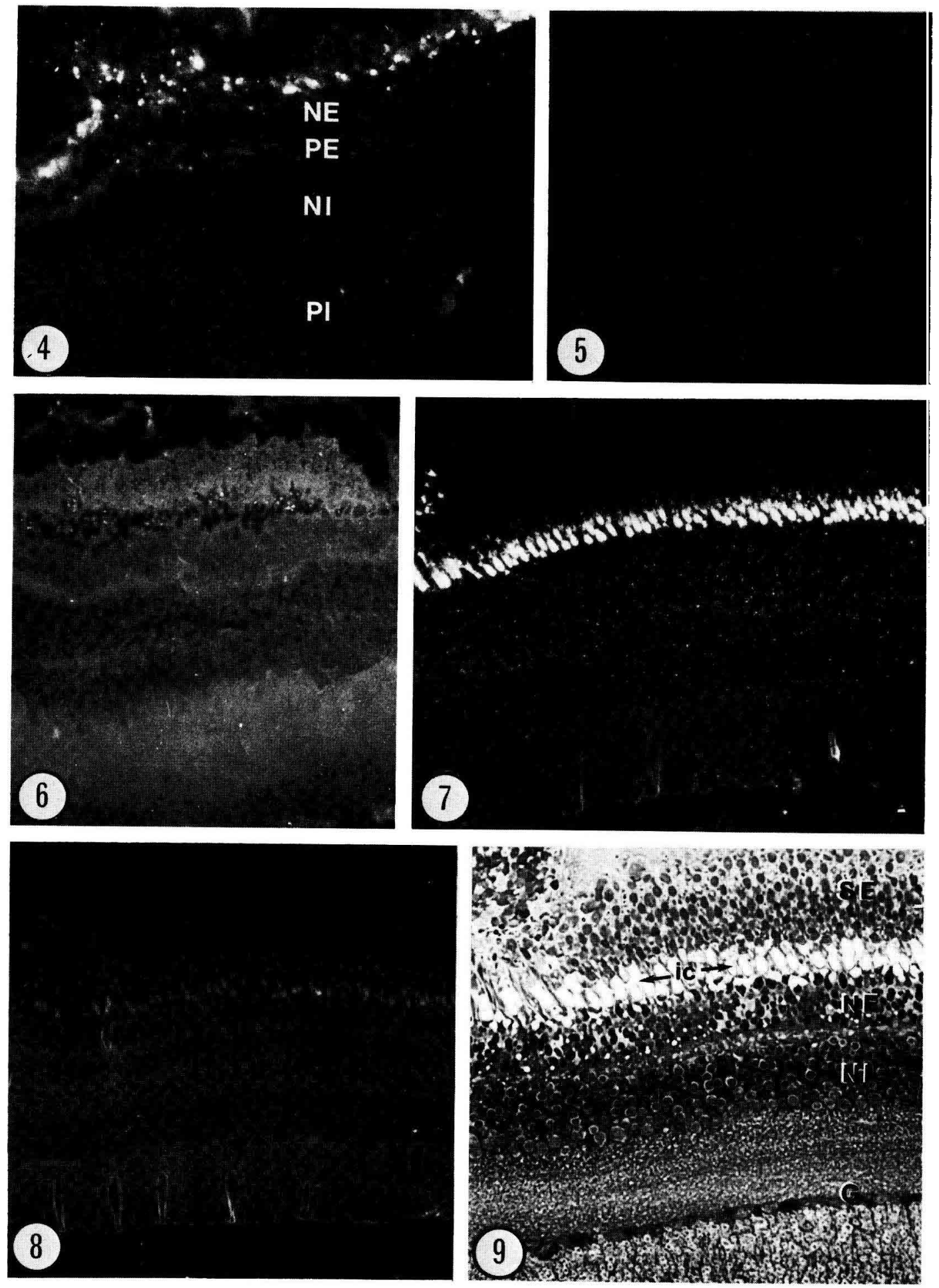
des photorécepteurs (face externe) (fig. 7). L'intensité des réactions diminue avec la concentration de l'anticorps mais une fluorescence spécifique est encore observable pour une dilution au $1 / 100^{\circ}$ du sérum natif. Les préparations témoins traitées avec le SLAC ne présentent pas de fluorescence spécifique dans la partie externe de la rétine (fig. 8 ).

Le contrôle sur des coupes colorées au bleu de toluidine montre que les réactions pourraient être superposées à des inclusions claires correspondant au segment interne des photorécepteurs (fig. 9).

\section{Discussion.}

\section{1. - Sur les variations du taux de mélatonine dans l'organe pinéal, la rétine et le plasma.}

Quelle que soit la saison, le taux de mélatonine épiphysaire présente des variations nycthémérales importantes montrant des valeurs minimales au cours de la phase claire et des valeurs maximales au milieu de la phase obscure. Ces résultats sont en accord avec ceux obtenus précédemment par le test biologique " mélanophore " chez cinq autres espèces d'Oiseaux (Ralph et al., 1967 ; Lynch, 1971 ; Binkley et al., 1973 ; Ralph et al., 1974). Les quantités de mélatonine évaluées dans l'épiphyse du Pigeon par dosage radioimmunologique sont inférieures à celles obtenues chez le Poulet et chez la Caille (Ralph, 1976).

Selon Binkley et al. (1975) les fluctuations nycthémérales du taux de mélatonine dans l'épiphyse seraient dépendantes du cycle de l'activité $\mathrm{N}$-acétyltransférasique (NAT). Néanmoins, compte tenu des résultats acquis chez l'Oiseau par d'autres auteurs, un cycle circadien de méthylation des hydroxindo-

\section{PLANCHE ।}

Localisation de la mélatonine par immunohistochimie dans la rétine de Pigeon.

FIGS. 4, 5, 6. - Rétine de Pigeon fixée à l'éthanol, après congélation. FIG. 4 : L'anticorps dirigé contre la mélatonine donne des réactions positives au-dessus de la couche nucléaire externe (NE). PE : couche plexiforme externe; $\mathrm{NI}$ : couche nucléaire interne ; $\mathrm{PI}$ : couche plexiforme interne. Anti-mélatonine $1 / 10^{\mathrm{e}} .(\times 300)$. FIG. 5 : Aucune réaction n'est observable sur les préparations témoin (SLAC 1/10 $).(\times 300)$. FIG. 6 : Absence de fluorescence chez un animal traité par la p-CPA. Anti-mélatonine $1 / 10^{\mathrm{e}} .(\times 180)$.

FIGS. 7, 8, 9. - Rétine de Pigeon fixée au glutaraldéhyde et inclus dans le GMA. FIG. 7: Une fluorescence intense est observable au-dessus de la couche nucléaire externe. Anti-mélatonine 1/100e. ( $\times 200)$. FIG. 8 : Aucune réaction n'apparaît sur les préparations témoin (SLAC 1/100e). $(\times 180)$. FIG. 9 : Structure de la rétine de Pigeon après coloration au bleu de toluidine. Les inclusions claires (ic) situées dans la couche des segments internes des photorécepteurs pourraient représenter les sites de fixation de l'anticorps anti-mélatonine. G : couche des cellules glanglionnaires; NE : couche nucléaire externe; NI : couche nucléaire interne ; SE : segments externes des photorécepteurs. $(\times 300)$. 
les faisant intervenir l'enzyme hydroxyindole-O-méthyltransférase (HIOMT) est à prendre en considération (Balemans et al., 1981). La mélatonine, synthétisée dans l'épiphyse, est essentiellement libérée dans le sang (Pang et Ralph, 1975). La mesure du taux de mélatonine plasmatique réalisée ici nous permet de déceler un pic au cours de la phase obscure qui pourrait être le reflet de l'augmentation de sa synthèse dans l'épiphyse (Pelham, 1975). Cependant une fraction de la mélatonine plasmatique peut également provenir d'autres organes ou tissus (exemple chez l'Oiseau : la rétine).

Malgré les conditions expérimentales sensiblement différentes, nous ferons observer que les cycles de la 5-HT et du 5-HIAA étudiés par Quay (1966) en photopériode $14 \mathrm{~L} / 10 \mathrm{D}$ et ceux de la mélatonine, déterminés ici, présentent des relations opposées de phase. Pour ces trois indoles, des données du même type ont été obtenues chez le Rat (Quay, 1974). On constate également, chez le Pigeon, que l'amplitude du cycle de la 5-HT est beaucoup plus élevée que celle de la mélatonine. Enfin la concentration minimale du 5-HIAA, supérieure au contenu maximal de mélatonine pourrait indiquer que la voie catabolique de la 5-HT reste prédominante en phase nocturne. Des résultats convergents ont été obtenus au cours de l'étude du métabolisme indolique dans l'organe pinéal de Pigeon isolé in vitro (Voisin, 1982 a).

L'étude des variations nycthémérales du taux de mélatonine dans l'épiphyse montre une amplitude plus importante du pic nocturne en juillet qu'en novembre. Nous ne retrouvons donc pas l'effet d'un changement artificiel du cycle d'éclairement rapporté par Ralph (1976) chez le Poulet, correspondant à un maintien de fortes concentrations de mélatonine en jours courts et de faibles concentrations en jours longs. A ce sujet, il convient de souligner que dans les conditions naturelles, le changement de saison ne se réduit pas aux modifications du rapport L/D mais entraîne la variation de nombreux facteurs exogènes (ex : intensité et spectre de la lumière incidente, température) pouvant intervenir comme synchroniseurs et éventuellement de facteurs endogènes liés à l'état physiologique des animaux. II faut noter également que nos résultats sont en accord avec ceux concernant les variations de l'activité HIOMT dans la production de certains méthoxyindoles (mélatonine/5-méthoxytryptophol : Balemans et al., 1981, chez la Perruche). Ces travaux relatent l'existence de pics nocturnes de l'activité HIOMT dont l'amplitude est plus élevée au printemps qu'en automne. Chez le Hamster, de récents travaux montrent que la synthèse de mélatonine est également maximale en été (Balemans et al. : communication personnelle). Les variations circannuelles de l'activité HIOMT, chez le Moineau, mesurées par Barfuss et Ellis (1971), donnent au contraire des valeurs plus élevées en novembre qu'en juillet. Toutefois, ces mesures ont été effectuées sur des animaux sacrifiés au cours de la photophase sans qu'il soit tenu compte des fluctuations nycthémérales de la synthèse de mélatonine.

Dans la rétine de Pigeon, l'augmentation de la concentration en mélatonine au cours de la phase nocturne pourrait refléter l'augmentation de l'activité NAT (Hamm et Menaker, 1980), alors que l'activité HIOMT ne semble pas présenter de variations nycthémérales dans la rétine pour la production de mélatonine (Wainwright, 1979 ; Pévet et al., 1980). 
2. - Sur l'identification par immunohistochimie des sites de stockage de la mélatonine dans la glande pinéale et la rétine.

Les résultats obtenus sur la rétine du Pigeon dans les différentes conditions expérimentales suggèrent que la mélatonine et/ou une molécule apparentée pourrait être stockée dans le segment interne des photorécepteurs et/ou dans les cellules de Müller. Ces résultats sont à rapprocher des observations effectuées chez des Poissons, Reptiles et Mammifères avec des anticorps dirigés contre la mélatonine obtenus à partir de différents immunogènes (Bubenik et al., 1974 ; Vivien-Roels et al., 1981 et réf.). La diminution importante de la fluorescence chez les animaux traités par la p-CPA permet d'avancer que ce sont bien des indoles qui sont reconnus au cours de la réaction. Un effet similaire du traitement à la p-CPA a été mis en évidence dans le colon de Rat (Holloway et al., 1980).

Par ailleurs, l'absence de réactions dans une structure riche en sérotonine comme l'organe pinéal du Pigeon (Quay, 1966 ; Voisin, 1982 a, b) constitue un élément pouvant indiquer que les molécules apparentées à la sérotonine (groupe des 5-hydroxyndoles) ne sont pas reconnues et que l'immunoréactivité concerne des molécules apparentées à la mélatonine (groupe des 5-méthoxyindoles). Les différences d'immunoréactivité observées entre l'épiphyse et la rétine du Pigeon doivent être interprétées en tenant compte des processus de fixation utilisés. La mélatonine étant très soluble dans l'éthanol, la fixation par cet alcool des coupes de tissu congelé pourrait entraîner l'extraction du méthoxyindole, dans le cas où son stockage ne ferait pas intervenir de liaison à une macromolécule.

Ainsi, nos résultats pourraient traduire le stockage de la mélatonine sous forme liée dans les photorécepteurs rétiniens (et/ou les cellules de Müller) et sous forme libre (?) dans l'épithélium épiphysaire (constitué de photorécepteurs modifiés et de cellules interstitielles : Voisin, 1982 a, b). Rappelons que la mélatonine ou une molécule apparentée a pu être visualisée en immunoultracytochimie dans les photorécepteurs typiques de l'épiphyse du Brochet (Falcon et al., 1981).

La mélatonine étant peu réactive avec le glutaraldéhyde, la rétention d'une forme libre de cet indole avant inclusion dans le glycolméthacrylate est incertaine et les mêmes remarques que celles présentées ci-dessus peuvent s'appliquer à ce type de préparation. De plus, la 5-méthoxytryptamine, après réaction avec le glutaraldéhyde, pourrait constituer un antigène reconnu par l'anticorps utilisé ici. Ainsi, dans ces conditions expérimentales, le composé immunoréactif pourrait correspondre à un " pool " de mélatonine stockée sous forme d'un complexe retenu par la fixation et éventuellement à la 5-méthoxytryptamine. De nouvelles recherches comparées sont actuellement entreprises afin d'aboutir à une interprétation plus précise des résultats actuels. 
Remerciements. - Nous remercions MM. les Dr. Balemans et Calas qui ont accepté de lire et amender cet article, M. le Dr. Falcon qui nous a initié et aidé dans la partie immunocytochimique, Mlle le Dr. M.-T. Juillard qui a contribué à la partie expérimentale de ce travail, et Mlle D. Decourt qui a assuré la dactylographie. Ce travail a pu être réalisé grâce à une bourse et un contrat DGRST $n^{\circ}$ 80-7-0339 et à l'appui financier du CNRS et de la Fondation Langlois.

\section{Références}

BALEMANS M. G. M., COLLIN J. P., LEGERSTEE W. C., JUILLARD M. T., 1981. Preliminary investigations on the circadian rhythmicity of the methylation of 5-hydroxyindoles in the pineal gland of the parakeet. Biol. cell., 42, 167-172.

BARFUSS D. W., ELLIS L. C., 1971. Seasonal cycles in melatonin synthesis by the pineal gland as related to testicular function in the house sparrow (Passer domesticus). Gen. comp. Endocrinol., 17, 183-193.

BINKLEY S., MacBRIDE S. E., KLEIN D. C., RALPH C. L., 1973. Pineal enzymes : regulation of avian melatonin synthesis. Science, 181, 273-275.

BINKLEY S., MacBRIDE S. E., KLEIN D. C., RALPH C. L., 1975. Regulation of pineal rhythms in chickens : refractory period and nonvisual light perception. Endocrinology, 96, 848-853.

BUBENIK G. A., BROWN G. M., UHLIR I., GROTA L. J., 1974. Immunohistological localization of $\mathrm{N}$-acetylindolealkylamines in pineal gland, retina and cerebellum. Brain Res., 81. 233-242.

COLLIN J. P., OKSCHE A., 1981. Structural and functional relationships in the nonmammalian pineal gland, 27-67. In REITER R. J., The pineal gland : anatomy and biochemistry, Vol. 1, CRC Press Inc., Boca Raton, Florida.

DEGUCHI T., 1979. Role of adenosine $3^{\prime}-5^{\prime}$ monophosphate in the regulation of circadian oscillation of serotonin $\mathrm{N}$-acetyltransferase activity in cultured chicken pineal gland. J. Neurochem., 33, 45-51.

FALCON J., GEFFARD M., JUILLARD M. T., DELAAGE M., COLLIN J. P., 1981. Melatonin-like immunoreactivity in photoreceptor cells. A study in the teleost pineal organ and the concept of photoneuroendocrine cells. Biol. cell., 42, 65-68.

GASTON S., MENAKER M., 1968. Pineal function : the biological clock in the sparrow ? Science, 160, 1125-1127.

GEFFARD M. R., PUIZILLOUT J. J., DELAAGE M. A., 1982. A single radio-immunological assay for Serotonin, N-Acetylserotonin and Melatonin. J. Neurochem., 39, 1271-1277.

GWINNER E., BENZINGER I., 1978. Synchronization of a circadian rhythm in pinealectomized european starlings by daily injections of melatonin. J. comp. Physiol., 127, 209-213.

HAMM H. E., MENAKER M., 1980. Retinal rhythms in chicks : circadian variation in melatonin and serotonin $\mathrm{N}$-acetyltransferase activity. Proc. nat. Acad. Sci. USA, 77, 4998-5002.

HOLLOWAY W. R., GROTA L. J., BROWN G. M., 1980. Determination of immunoreactive melatonin in the colon of the rat by immunocytochemistry. J. Histochem. Cytochem., 28, 255-262.

KOE B. K., WEISSMAN A., 1966. p-chlorophenylalanine : a specific depletor of brain serotonin. J. Pharmacol. exp. Therap., 154, 499-516.

LEDUC E. H., BERNHARD W., 1967. Recent modifications of the glycol methacrylate embedding procedure. J. U/trastructure Res., 19, 196-199.

LOWRY O. H., ROSEBROUGH N. J., FARR A. L., RONDAL R. J., 1951. Protein measurements with the folin phenol reagent. J. Biochem., 193, 265-275.

LYNCH H. J., 1971. Diurnal oscillations in pineal melatonin content. Life Sci., 10, 791-795.

PANG S. F., RALPH C. L., 1975. Mode of secretion of pineal melatonin in the chicken. Gen. comp. Endocrinol., 27, 125-128.

PELHAM R. W., 1975. A serum melatonin rhythm in chickens and its abolition by pinealectomy. Endocrinology, 96, 543-546. 
PÉVet P., BAlemans M. G. M., Legerstee W. C., VIVIEN-Roels B., 1980. Circadian rhythmicity of the activity of hydroxyindole-O-methyltransferase (HIOMT) in the formation of melatonin and 5-methoxytryptophol in the pineal, retina and harderian gland of the golden hamster. J. neural Transm., 49, 229-245.

QUAY W. B., 1966. Rhythmic and light- induced changes in levels of pineal 5-hydroxyindoles in the pigeon (Columba livia). Gen. comp. Endocrinol., 6, 371-377.

QUAY W. B., 1974. Pineal chemistry in cellular and physiological mechanisms. Thomas, Springfield, pp. 430.

RALPH C. L., 1976. Correlations of melatonin content in pineal gland, blood, and brain of some birds and mammals. Amer. Zool., 16, 35-43.

RALPH C. L., 1981. The pineal and reproduction in birds, 31-43. In REITER R. J., The pineal gland : reproductive effects, Vol. 2. CRC Press, Inc. Boca Raton, Florida.

RALPH C. L., HEDLUND L., MURPHY W. A., 1967. Diurnal cycles of melatonin in bird pineal bodies. Comp. Biochem. Physiol., 22, 591-599.

RALPH C. L., PELHAM R. W., MacBRIDE S. E., REILLY D. P., 1974. Persistent rhythms of pineal and serum melatonin in cockerels in continuous darkness. J. Endocr., 63, 319-324.

ROLLAG M. D., 1981. Methods for measuring pineal hormones, 273-302. In REITER R. J., The pineal gland: anatomy and biochemistry, Vol. 1, CRC Press Inc., Boca Raton, Florida.

VIVIEN-ROELS B., PÉVET P., DUBOIS M. P., ARENDT J., BROWN G. M., 1981. Immunohistochemical evidence for the presence of melatonin in the pineal gland, the retina, and the harderian gland. Cell Tissue Res., 217, 105-115.

VOISIN P., 1982 a. Les cellules de la lignée réceptrice dans l'épiphyse aviaire et le problème des horloges cellulaires (étude immunologique, autoradiographique et radiobiochimique). Th. $3^{e}$ cycle, $n^{\circ} 839$, Univ. Poitiers.

VOISIN P., 1982 b. Etude de l'épithélium épiphysaire du pigeon dans les conditions naturelles et in vitro: ultrastructure et mise en évidence histochimique d'hydroxyindoles dans les cellules de la lignée réceptrice. Arch. Anat. micr. Morph. expér. (sous presse).

WAINWRIGHT S. D., 1979. Development of hydroxyindole-O-methyltransferase activity in the retina of the chick embryo and young chick. J. Neurochem., 32, 1099-1101.

ZIMMERMAN N. H., MENAKER M., 1979. The pineal gland : a pacemaker within the circadian system of the house sparrow. Proc. nat. Acad. Sci. USA, 76, 999-1003. 